\title{
No evidence from complementary data sources of a direct projection from the mouse anterior cingulate cortex to the hippocampal formation
}

\author{
Lilya Andrianova ${ }^{1,2}$, Steliana Yanakieva ${ }^{3}$, Gabriella Margetts-Smith ${ }^{1}$, Shivali Kohli ${ }^{1}$, Erica S \\ Brady $^{1}$, John P Aggleton ${ }^{3}$, and Michael T Craig ${ }^{1,2}$
}

1. Institute of Biomedical and Clinical Science, University of Exeter Medical School, Prince of Wales Road, Exeter, EX4 4PS, England, UK

2. School of Psychology \& Neuroscience, College of Medical, Veterinary and Life Sciences, University of Glasgow, Glasgow G12 8QQ, Scotland, UK.

3. School of Psychology, Cardiff University, 70 Park Place, Cardiff, CF10 3AT, Wales, UK

Correspondence to mick.craig@glasgow.ac.uk 


\begin{abstract}
The connectivity and interplay between the prefrontal cortex and hippocampus underpin a number of key cognitive processes, with changes in these interactions being implicated in both neurodevelopmental as well as neurodegenerative conditions. Understanding the precise cellular connections through which this circuit is organised is, therefore, vital for understanding these same processes. Overturning earlier findings, a recent study described a novel excitatory projection from anterior cingulate cortex to hippocampus. We sought to validate this unexpected finding using multiple, complementary methods: anterograde and retrograde anatomical tracing, using both anterograde and retrograde $A A V s$ and monosynaptic rabies tracing. Additionally, an extensive data search of the Allen Projection Brain Atlas database was conducted to find the stated projection within any of the deposited anatomical studies, as an independent verification of our own results. However, we failed to find any evidence of a direct, monosynaptic projection from mouse anterior cingulate cortex to the hippocampus proper.
\end{abstract}

\title{
Introduction
}

Models of the functional interactions between the rodent prefrontal cortex and the hippocampal formation have had to address their asymmetrical relationship. The subiculum and CA1 give rise to direct efferents that terminate in the prelimbic, infralimbic, and medial orbital cortices (Cenquizca and Swanson, 2007; Groenewegen et al., 1987; Jay and Witter, 1991; Swanson, 1981) as well as light projections to the anterior cingulate cortex (Cenquizca and Swanson, 2007; Jay and Witter, 1991). In contrast, direct projections from the prelimbic and anterior cingulate cortices to the CA fields or the subiculum are not observed (Jones and Witter, 2007; Segal and Landis, 1974; Sesack et al., 1989; Vogt and Miller, 1983). Meanwhile, only a very sparse projection from infralimbic cortex to CA1 has been described (Hurley et al., 1991), with a recent preprint providing evidence of long-range inhibitory projections from prelimbic and infralimbic cortices to hippocampus proper (Malik et al., 2021). For this reason, models of prefrontal 
regulatory action upon the hippocampus have emphasised the importance of indirect routes. These routes include relays via parahippocampal cortices and via subcortical sites such as nucleus reuniens and the anterior thalamic nuclei (Eichenbaum, 2017; Furtak et al., 2007; Jones and Witter, 2007; Prasad and Chudasama, 2013).

This relationship was transformed by the description of light, direct projections from the dorsal anterior cingulate cortex to the CA1 and CA3 fields in mice (Rajasethupath et al., 2015). Despite their sparsity, optogenetic manipulation of these same projections was sufficient to elicit contextual memory retrieval, and optogenetic stimulation of these axons evoked robust excitatory post-synaptic currents in pyramidal cells in both CA3 and CA1 (Rajasethupath et al., 2015). These findings are striking, not least because by revealing a previously unknown connection in the rodent brain, they suggest that there are potentially many such functional connections still waiting to be uncovered.

Given the impact of their functional findings on anterior cingulate efferents (Rajasethupath et al., 2015), the present study re-examined the status of the projections from the anterior cingulate area (ACA) to the hippocampal formation (dentate gyrus, CA fields, and subiculum) in mice. Anterograde tracing using adeno-associated viral vectors (AAVs) found no evidence of the anterior cingulate to hippocampus projection in mice, nor did rabies virus-assisted monosynaptic retrograde tracing from hippocampal pyramidal cells. Furthermore, a thorough data mining analysis of Allen Expression data also revealed no evidence of an anterior cingulate to HPC projection in mice.

\section{Methods}

All UK-based research was carried out in accordance with the UK Animals (Scientific Procedures) Act 1986 and was subject to local ethical review by the Animal Welfare and Ethical Review Board 
at the University of Exeter. All animals were maintained on a $12 \mathrm{~h}$ constant light / dark cycle and had access to food and water ad libitum. We used standard enrichment that included cardboard tubes, wooden chew blocks and nesting material.

\section{AAV anterograde and retrograde tracing}

This tracing study used adult C57BL/6 or SST-Cre mice of both sexes aged 2 to 5 months, ranging from $22.2 \mathrm{~g}$ to $31.3 \mathrm{~g}$ (mean age 3 months, mean weight $25 \mathrm{~g}$ ). To target the ACA, we made stereotaxic injections of $250 \mathrm{nl}$ of AAV5-CamKII-GFP $5.3 \times 10^{12} \mathrm{vg} / \mathrm{ml}$ (Viral Vector Facility, Neuroscience Centre Zurich, Swizterland) or AAV5/2-hSyn1-ChR2_mCherry $7.1 \times 10^{12} \mathrm{vg} / \mathrm{ml}$ (Viral Vector Facility, Neuroscience Centre Zurich, Switzerland). Briefly, mice were anaesthetised with $5 \%$ isoflurane, were placed on a heated pad for the duration of the surgery, and maintained at 1.5 to $2.5 \%$ isoflurane (with a flow rate of $\sim 2 \mathrm{Lmin}^{-1} \mathrm{O}_{2}$ ). Mice were given $0.1 \mathrm{mg} / \mathrm{kg}$ of buprenorphine (buprenorphine hydrochloride, Henry Schein) subcutaneously at the start of surgery as an adjunct analgesic, and carprofen (Rimadyl, Henry Schein) was given at a dose of $5 \mathrm{mg} / \mathrm{kg}$ subcutaneously at the end of surgery and on subsequent days, as required. An incision was made down the midline and a craniotomy was performed to allow injection of virus (250 nl). For anterograde tracing, we first used the co-ordinates reported by Rajasethupathy et al., 2015: A/P +1.0 mm (relative to Bregma), M/L $-0.35 \mathrm{~mm}$ and D/N-1.2 mm (from pia). Additionally, to minimise spread of virus into $M 2$ cortical area, we also injected at $A / P+1 \mathrm{~mm}$ (relative to Bregma), M/L $-0.2 \mathrm{~mm}$ and $\mathrm{D} / \mathrm{V}-1.3 \mathrm{~mm}$ (from pia) on the same rostrocaudal plane, and targeted ACA at a more rostral site A/P +1.75 mm (relative to Bregma), M/L -0.2 mm and D/V-1.5 mm (from pia). For retrograde tracing with AAVs, we used rAAV-retro, developed at HHMI Janelia Campus (Tervo et al., 2016), and injected rAAV-retro/2-CAG-EGFP_Cre-WPRE-SV40p(A) $5.9 \times 10^{12} \mathrm{vg} / \mathrm{ml}$ (Viral Vector Facility, Neuroscience Centre Zurich, Switzerland) into hippocampal region CA1, using the following co-ordinates: A/P $-2 \mathrm{~mm}$ (relative to Bregma), $\mathrm{M} / \mathrm{L}-1.5 \mathrm{~mm}$ and $\mathrm{D} / \mathrm{V}-1.35 \mathrm{~mm}$ (from pia) After surgical repair of the wound, mice were given $5 \mathrm{mg} / \mathrm{kg}$ carprofen (Rimadyl, Henry Schein) 
subcutaneously immediately after surgery, and again the following day. Further analgesia was provided as required. The mice were maintained for at least 3 weeks to provide sufficient time to expression of virally-delivered transgenes, and were killed by transcardial perfusion / fixation with 4\% paraformaldehyde (Sigma-Aldrich, UK) in $0.1 \mathrm{M}$ phosphate buffer.

Perfused brains were cryoprotected with $30 \%$ sucrose solution (Fisher Schientific, UK) and sliced at $30 \mu \mathrm{m}$ using a SM2010R freezing microtome (Leica, UK). The slices were stained with DAPI (HelloBio, UK) and fluorescence was visualised using CoolLED pE-4000 (CoolLED, UK); photos taken on Nikon 800 microscope. For anterograde tracing experiments 32 mice were injected (2 were excluded due to failed injections as no fluorescence was observed) and 10 mice were injected with a retrograde virus (2 mice were excluded due to failed injections). Viral expression patterns in individual mice at the injection sites of both sets of coordinates were overlayed (figure $1 A-B)$ to show the variation in the injections and typical spread of the virus in the injection areas. A qualitative assessment of the projection patterns was performed and representative images of regions of interest are presented on figure $1 \mathrm{C}-\mathrm{I}$.

\section{Monosynaptic Retrograde tracing}

For monosynaptic rabies tracing, we used methods reported by others (Sun et al., 2014). Adult Emx1-cre mice (Guo et al., 2000) were crossed with floxed TVA mice (Seidler et al., 2008) to allow specific targeting of pyramidal cells; Emx1-cre mice alone were used as controls to ensure the rabies virues did not transduce neurons in the absence of TVA gene. Mice of both sexes aged 3 to 7 months, ranging from $21.7 \mathrm{~g}$ to $40.6 \mathrm{~g}$ (mean age 4.5 months, mean weight $27.2 \mathrm{~g}$ ). To target the efferent projections to dCA1, we made stereotaxic injections of AAV8-FLEX-H2B-GFP2A-oG, titre $3.93 \times 10^{12} \mathrm{vg} / \mathrm{ml}$ (Salk Institute Viral Vector Core) followed by injection EnvA Gdeleted Rabies-mCherry $6.13 \times 10^{8} \mathrm{vg} / \mathrm{ml}$ (Salk Institute Viral Vector Core or Charité Universitätsmedizin Berlin Viral Vector Core) 2 weeks after the initial viral injection. Briefly, mice 
were anaesthetised with $5 \%$ isoflurane, were placed on a heated pad for the duration of the surgery, and maintained at 1.5 to $2.5 \%$ isoflurane (with a flow rate of $\sim 2 \mathrm{Lmin}^{-1} \mathrm{O}_{2}$ ). Mice were given $0.1 \mathrm{mg} / \mathrm{kg}$ of buprenorphine (buprenorphine hydrochloride, Henry Schein) subcutaneously at the start of surgery as an adjunct analgesic, and carprofen (Rimadyl, Henry Schein) was given at a dose of $5 \mathrm{mg} / \mathrm{kg}$ subcutaneously at the end of surgery and on subsequent days, as required. An incision was made down the midline and a craniotomy performed to allow injection of virus $(250 \mathrm{nl})$. We targeted hippocampal region CA1 at dorsal and ventral points, using the following co-ordinates: A/P -2 mm (relative to Bregma), $\mathrm{M} / \mathrm{L}-1.5 \mathrm{~mm}$ and $\mathrm{D} / \mathrm{V}-1.35 \mathrm{~mm}$ (from pia) and A/P $-2.8 \mathrm{~mm}$ (relative to Bregma), M/L -2.4 mm and D/V-4.2 mm (from pia) for dorsal and ventral CA1 regions respectively. After surgical repair of the wound, mice were given $5 \mathrm{mg} / \mathrm{kg}$ carprofen (Rimadyl, Henry Schein) subcutaneously immediately after surgery, and again the following day. Further analgesia was provided as required. The mice were maintained for 2 weeks to provide optimal time for expression, and were killed by transcardial perfusion / fixation with $4 \%$ paraformaldehyde (Cat number P6148 Sigma-Aldrich, UK) in $0.1 \mathrm{M}$ phosphate buffer.

Perfused brains were cryoprotected with $30 \%$ sucrose solution (Fisher Schientific, UK) and sliced at $50 \mu \mathrm{m}$ using a SM2010R freezing microtome (Leica, UK). The slices were mounted using HardSet Mounting Medium with DAPI (Vector Labs) and fluorescence was visualised using CoolLED; photos taken on Nikon 800 microscope. A total of 18 mice including 4 controls were injected ( 2 were excluded due to failed injections as no fluorescence from both viral constructs was observed). Representative images of the spread of the viruses can be found in figure 2. A semi-quantitative assessment of the projection patterns was performed by recording the number of slices with the cells expressing the virus in each region of interest present and the summary of this data is shown (figure $2 \mathrm{P}$ ). These monosynaptic rabies tracing data were generated in the same experiment that we have reported elsewhere, focusing on connections between nucleus reuniens and CA1 pyramidal cells (Andrianova et al., 2021). 


\section{Allen Projection Atlas Data Mining}

The Allen Mouse Brain Connectivity Atlas (http://mouse.brain-map.org/) is a comprehensive database of images of axonal projections in the mouse brain. To create the atlas, each mouse brain is injected with enhanced green fluorescent protein (EGFP) expressing adeno-associated virus (AVV) as an anterograde tracer into a source brain region (for further details see http://connectivty.brain-mpa.org). Then, the axonal projections are systematically imaged using a TisseCyte 1000 serial two-photon tomography system. The data in the atlas were collected from adult mice on postnatal day P56 \pm 2 (for details, see Oh et al., 2014). Each case in the atlas contains high resolution images and quantified projection information based on the optical density of label. Detailed histograms of the signal in each structure are presented giving the projection volume $\left(\mathrm{mm}^{3}\right)$ and projection density (the fraction of area occupied by a fluorescent signal from the viral construct used relative to the whole structure). Details of the algorithms used are provided (Kuan et al., 2015). The sensitivity of this viral tracer has been compared with biotinylated dextran amine (BDA), revealing comparable transport properties (http://connectivty.brain-mpa.org).

To establish whether there are direct projections from the anterior cingulate area (dorsal ACA and ventral ACA) to the hippocampal formation [Cornu Ammonis (CA) 1-3, dentate gyrus (DG), and subiculum (SUB)], a comprehensive systematic search was completed. In addition, the density of fibre label in the cingulum bundle (CB) was taken from the Allen Atlas. The dorsal and ventral ACA were entered as a source structure and the CA1-3, DG, SUB, and cingulum bundle as target structures. The search was further filtered for: (1) Reporter type: EGFP; (2) Hemisphere: Either; (3) Minimum target injection volume: $0.0001 \mathrm{~mm}^{3}$. A total of 99 cases were returned. Then, 63 cases with injection volumes of $0.02-0.3 \mathrm{~mm}^{3}$ in the ACA were selected for further processing, 
of which 47 had a minimum of $90 \%$ of the injected virus (mean $=0.108 \mathrm{~mm}^{3} ; S D=0.059 \mathrm{~mm}^{3}$ ) within the dorsal and ventral anterior cingulate cortices. Table 1 lists all 47 cases and provides further information about the transgenic lines and the individual injections. The large majority ( $\mathrm{n}$ $=44$ ) of the injections were made in the right hemisphere, the remaining three in the left. In the few cases where some signal was reported in areas CA1 and CA3, we used the Willcoxon signed rank test to compare that signal with the corresponding signal in the ipsilateral DG and CA2 to help test for background variation. (The dentate gyrus and CA2 were selected as there are no reports of ACA inputs to these subareas.)

There were three cases of wild-type mice (C57BL/6J) with $100 \%$ of the injection volume within ACA. Of the 44 transgenic mice, 28 had $100 \%$ of the tracer within the ACA, making a total of 31 such cases. The other 16 cases had a minimum of $90 \%$ of the tracer in the ACA and the remainder in adjacent areas, including the secondary motor area, the prelimbic cortex, and adjacent fibre tracts in various proportions. These 16 cases were, therefore, not considered in the initial analyses. In the WT group all mice were male, whilst in the transgenic group of 28 cases with $100 \%$ of the injection in the ACA, 12 were female. In the same transgenic group, 27 of the 28 injections were in the right hemisphere (see Table 1 for a list of included studies).

\section{Results}

\section{Anterograde virus-assisted tracing}

We carried out stereotaxic injections of AAV vectors to allow expression of GFP or mCherry in ACA neurons to allow us to map their projections (figure 1). We targeted our injections into ACA / mPFC at two different AP levels: $1.0 \mathrm{~mm}$ rostral to Bregma (figure $1 \mathrm{~A}$ ) and $1.75 \mathrm{~mm}$ rostral to Bregma (figure 1B). The more caudal co-ordinates exactly matched those reported by Rajasethupath and colleagues (2015), which also produced some somatic labelling in M2. We then targeted a site deeper and slightly more lateral within a more rostral point in the rostrocaudal 
axis, which led to somatic labelling restricted entirely within the subdivisions of the prefrontal cortex. In all cases, we saw prefrontal efferents within retrosplenial cortex and several thalamic nuclei, as expected, but found no evidence of axons in any hippocampal subfield or subiculum (figure $1 \mathrm{C}, \mathrm{E}, \mathrm{I})$. A summary of the projections from our anterograde labelling experiment is presented in supplementary Table 1, which incorporates data from a total of 30 mice. Supplementary figure 1 presents more examples from the anterograde tracing experiments.

\section{Monosynaptic retrograde tracing}

After examining with anterograde virus-assisted tracing, we next used mononsynaptic rabiesassisted viral tracing to determine whether pyramidal cells in hippocampal region CA1 received direct inputs from ACA (experimental protocol summarised in figure 2A). We carried out tracing from both dorsal CA1 (dCA1; $n=6$ mice; figure 2B - C) and ventral CA1 (vCA1; $n=4$ mice; figure $2 \mathrm{l}-\mathrm{J}$ ). Acting as positive controls, and as we reported previously (Andrianova et al., 2021), retrogradely-labelled neurons were detected in all brain regions in all mice that one would expect to project to CA1: both dCA1 and vCA1 received monosynaptic inputs from medial vCA1 (figure 2M). As reported by others (Sun et al., 2014), we found evidence of monosynaptic inputs to dCA1 from dorsal subiculum (figure $2 \mathrm{~F}$ ) and to vCA1 from ventral subiculum, although we did not determine whether these neurons were GABAergic or glutamatergic. As expected, vCA1, but not dCA1, received monosynaptic input from the amygdala (figure $2 \mathrm{~L}$ ). Importantly, however, we found no evidence of monosynaptic projections from ACA or any other prefronal region to either dCA1 (figure 2D, G-H) or vCA1 (figure $2 \mathrm{~K}, \mathrm{~N}-\mathrm{O}$ ). These data are summarised in figure $2 \mathrm{P}$. 

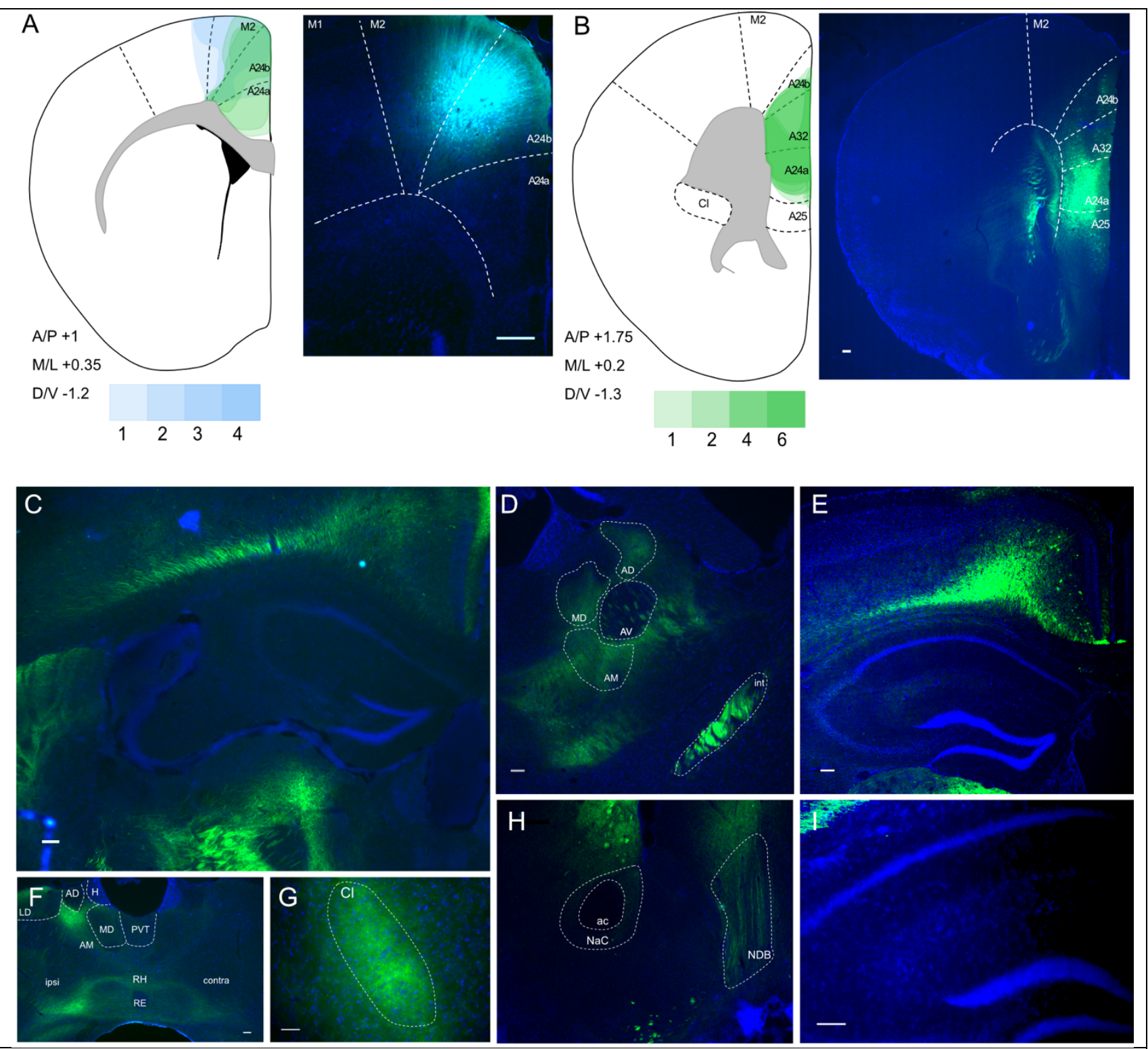

Figure 1: Anterograde viral tracing from ACA CaMKII-expressing neurons shows no excitatory projection to the dCA1 region of hippocampus. (A) Prefrontal cortex injection sites and spread, blue overlays show injections made using previously published coordinates (Rajasethupathy et al., 2015), green overlays show in-house initial optimization attempt, $n=3$ and $n=3$ respectively, (B) Prefrontal cortex injection site and spread of individual injections using inhouse optimized coordinates; each overlay shape represents one injection, $n=7$. (C) Representative images of the regions of interest and known target regions of the PFC, (D) ipsilateral thalamic nuclei and internal capsule, (E) dorsal hippocampus shows no fibres, unlike the overlaying RSC and other cortices, (F) rostral and midline thalamus in both ipsi and contralateral hemispheres, showing both fibre and terminal label, (G) fibres in ipsilateral claustrum, $(\mathrm{H})$ fibres are found in diagonal band nucleus and striatum, but absent in nucleus accumbens, (I) higher magnification image of dorsal CA1 region of the hippocampus, showing no fibres. A24a - area 24a (infralimbic), A24b - area 24b (cingulate cortex 1), A25 - area 25 (dorsal peduncular cortex), A32 - area 32 (prelimbic), ac - anterior commissure, ACA - anterior cingulate area, AD - anterodorsal nucleus, AM - anteromedial nucleus, AV - anteroventral nucleus, $\mathrm{Cl}$ - 
bioRxiv preprint doi: https://doi.org/10.1101/2022.01.25.477805; this version posted January 26, 2022. The copyright holder for this preprint (which was not certified by peer review) is the author/funder, who has granted bioRxiv a license to display the preprint in perpetuity. It is made available under aCC-BY-NC 4.0 International license.

claustrum, $\mathrm{H}$ - habenula, int - internal capsule, LD - lateral dorsal nucleus, M1 - primary motor area, M2 - secondary motor area, MD - mediodorsal nucleus, $\mathrm{NaC}$ - nucleus accumbens core area, NDB - diagonal band nucleus, PVT - paraventricular nucleus of the thalamus, RE nucleus reuniens, $\mathrm{RH}$ - rhomboid nucleus. Scale bar 100 microns 


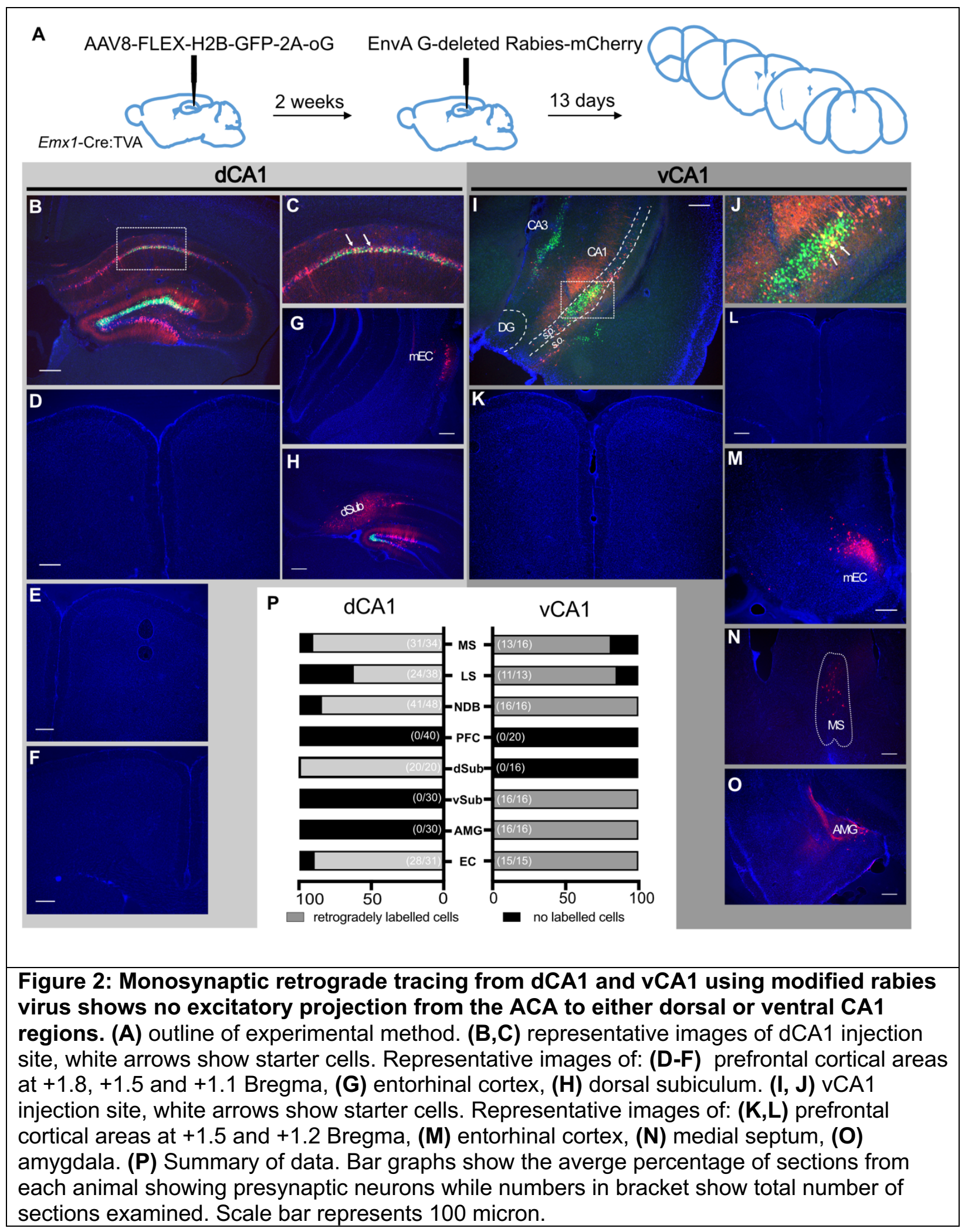




\section{Allen Atlas Data}

A total of 31 cases had viral injections confined within the ACA. For these cases we recorded the optical density measures for transported fluorescent fibres in the regions of interest. The medians (Mdn) and ranges for each hippocampal region by group (WT or transgenic) are presented in figure 3. In the WT group (figure $3 A$ and D) there was no evidence of signal in either the ipsilateral or contralateral CA1 field (all optical density measures = zero), and only one case contained any signal in the ipsilateral CA3 field (0.0002). For the transgenic group (figure 3B - D), the median signal for CA3 and CA1 remained at zero, but there were eight cases with potential signal in the ipsilateral and/or the contralateral CA1 and/or CA3 fields (all <0.0007). However, in all of these cases there was a low, but variable, background signal. For this reason, we compared the signal in areas CA1 and CA3 (which have potential anterior cingulate inputs) with that in the DG and area CA2 (neither of which are thought to receive anterior cingulate inputs). To maximise the likelihood of finding evidence for a projection we just used the eight cases with possible CA3 or possible CA1 label for the statistical comparisons.

There was no evidence that either CA1 or CA3 contained higher signal levels in these eight cases, i.e., the higher optical measures reflected a higher background signal. Wilcoxon signed rank tests found no statistical difference in the signal between the ipsilateral DG and CA3 $(z=-0.845, p=$ 0.388), CA2 and CA3 ( $z=-0.510, p=0.610)$, or the contralateral DG and CA3 $(z=-0.755, p=0.438)$, and contralateral CA2 and CA3 $(z=-1.841, p=0.066)$. Likewise, there were no significant differences between the signal in ipsilateral CA2 and CA1 $(z=-1.63, p=0.102)$ or the contralateral CA2 and CA1 ( $z=-1.07, p=0.285)$. While there were significant differences between both the ipsilateral DG and CA1 ( $z=-2.4, p=0.016)$ and the contralateral DG and CA1 $(z=-2.2, p=0.028)$, inspection of the data showed that in seven of the cases the signal was higher in the DG than CA1, i.e., the opposite direction to that consistent with an ACA projection to CA1. 
In contrast, the signal in the cingulum bundle (both ipsilateral and contralateral) was higher in 29 of the 31 cases than the signal in CA1-CA3 and the DG. In the WT group the median signal density in the ipsilateral $C B$ was 0.031 and 0.0014 in the contralateral $C B 0.014$ (see figure 3Ei for ranges). Similarly, the transgenic group with right-hemisphere injections had a median signal density in the ipsilateral $\mathrm{CB}$ of 0.0043 and 0.0002 in the contralateral $\mathrm{CB}$ (figure 3Ei). The presence of $\mathrm{CB}$ fibre label helps to confirm the effective transport of the tracer from the injection site. Lastly, there was no evidence of a projection from the ACA to the subiculum in any group as the median signal density observed for the ipsilateral and contralateral subiculum was a maximum of 0.0001 (Figure 3Eii).

The median density signal in the 16 transgenic cases with injection volumes of over $90 \%$ within the ACA was consistent with the data from the other 28 transgenic cases (see supplementary Table 1). The median group signal in both CA1 and CA3 was zero, with 10 cases having potential evidence of a signal $(\max =0.0007)$ in CA1 and/or CA3. Again, Wilcoxon signed rank tests revealed no evidence of a statistical difference in the signal between the ipsilateral or contralateral DG and CA3 or CA2 and CA3 (all $z<-1.63$, all $p_{\mathrm{s}}>0.102$ ) nor between the ipsilateral and contralateral CA2 and CA1 (all $z<-1.34$, all $p_{\mathrm{s}}>0.180$ ). There were, however, statistically significant differences between both the ipsilateral DG and CA1 $(z=-2.56, p=.010)$ and the contralateral DG and CA1 $(z=-2.03, p=.042)$, but again the higher signal was in the DG. Further inspection of individual cases showed that one case (ID:125801033) had noticeably higher signal density in the ipsilateral DG (0.0108) and SUB (0.0039). A second case (ID: 585911240) had the highest signal density in the ipsilateral CA3 (0.0007). However, in both cases $5 \%$ of the injection had leaked into adjacent fibre tracts. 


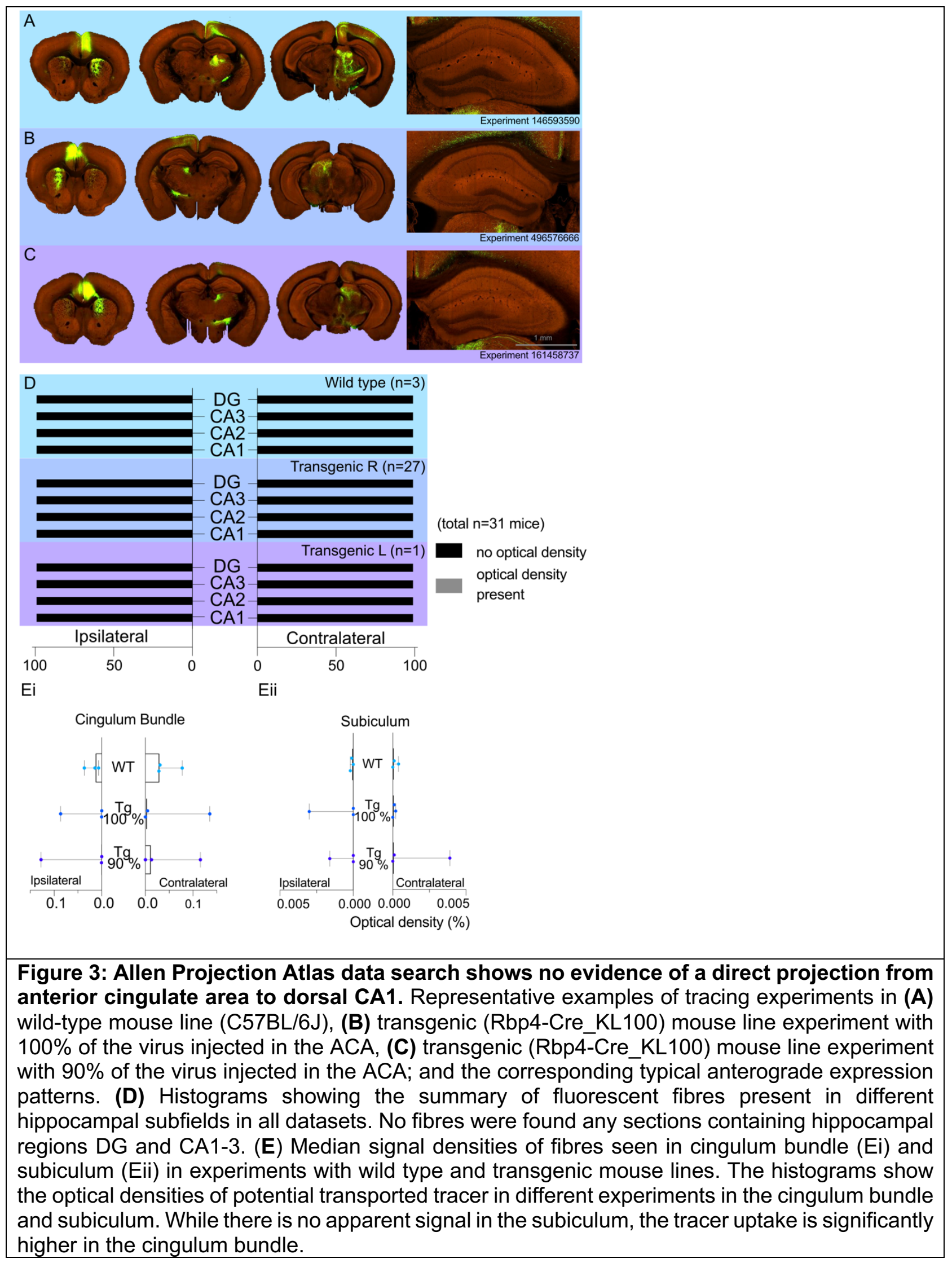


Of concern was whether the adjacent cortical field M2 might project to the hippocampal formation and, thereby, be a potential confound for tracer injections that extended into this area. For this reason we examined the Allen Mouse Brain Connectivity Atlas (http://mouse.brain-map.org/) for wild type cases meeting the inclusion criteria with injections of $90 \%$ or more within area M2. Any case in which the tracer extended into the anterior cingulate cortices was excluded. The search returned only one such case (ID: 585025284), which showed no evidence of a projection to CA1, CA2, or subiculum (all signal density counts zero). There was a very low signal count within the ipsilateral CA3 and DG (<0.0002). In an additional case (ID:141602484) the viral injection involved both the primary motor cortex (46\%) and area M2 (54\%). The only hippocampal signal was in the ipsilateral CA3 $(<0.0002)$. Both cases had appreciably more signal in the ipsilateral than contralateral cingulum bundle, consistent with viable transport.

\section{$\underline{\text { Retrograde tracing using a single virus strategy }}$}

Thus far, we have failed to detect evidence of a direct projection from the cingulate cortex to hippocampus. The question remains of why there is a discrepancy between our data and that reported previously (Rajasethupathy et al., 2015). In our rabies-virus assisted retrograde tracing experiment, we used a pseudotyped glycoprotein-deleted rabies virus that could only infect neurons expressing TVA, and could only spread transynaptically from neurons that also expressed the rabies glycoprotein, delivered via a secondary helper virus, as described elsewhere (Sun et al., 2014). In contrast, Rajasethupathy et al. (2015) used a non-pseudotyped glycoproteindeleted rabies virus which acts as a first-order tracer from the injection site (Wickersham et al., 2007). To test whether this strategy could provide off-target retrograde labelling, we made stereotaxic injections of a retrograde AAV vector (Tervo et al., 2016) into dorsal CA1 (figure 4A). Using this approach, in addition to dense neuronal labelling of CA1 pyramidal cells (Figure 4B), we saw labelling in the entorhinal cortex (figure 4F and 4G), as would be expected. However, similar to the extended figure 1 of (Rajasethupathy et al., 2015), we saw labelling of neurons 
directly above the injection site, and throughout both superficial and deep cortical layers in much of the brain (figure 4B; see also Extended Data figure 1a of Rajasethupathy et al., 2015). Additionally, we observed dense labelling of neurons in the anterior thalamic nuclei (figure 4E), which do not project to hippocampus proper (Mathiasen et al., 2020) and, importantly, we saw bilateral labelling in multiple subdivisions of prefrontal cortex, including the anterior cingulate cortex; it was most dense in the ipsilateral hemisphere (figure 4C). Without the selectivity of the pseudotyped rabies tracing approach from specific neuronal populations, we believe that the offtarget retrograde labelling in this region was due to uptake of virus in axons passing through the cingulum and / or corpus callosum as well as along the path of the injection needle used.

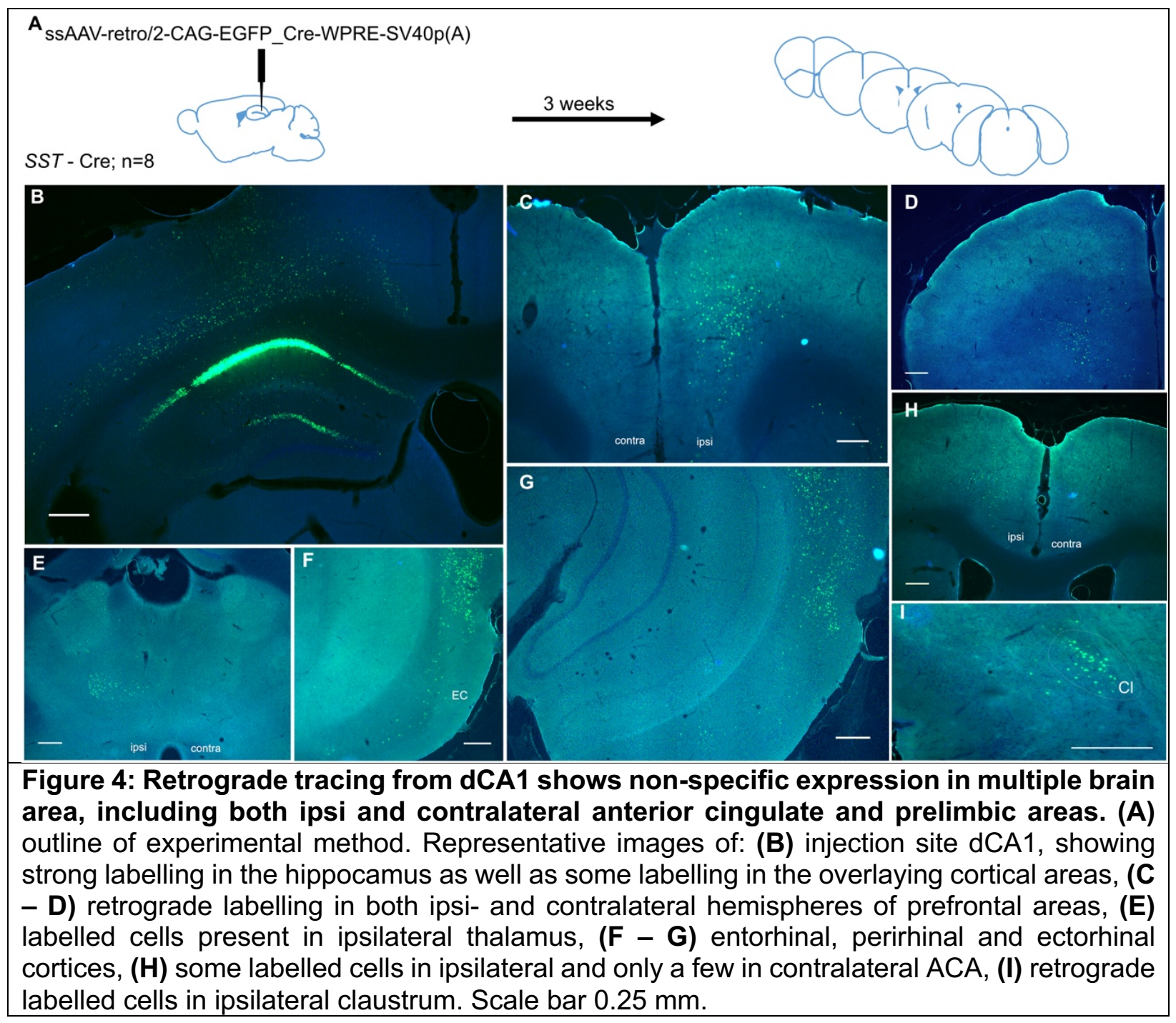




\section{Discussion}

Here, we attempted to replicate the report of a novel projection from anterior cingulate cortex to hippocampus (Rajasethupathy et al., 2015) but failed to find any evidence of this projection in the mouse after examining a combination of tracing methods. We have also presented some technical issues that may have led to a misleading interpretation of the data in the original publication. We believe that it is important to report this failure to replicate, given that the existence of a direct anterior cingulate cortex projection to the hippocampus was published in a highly influential journal and, at the time of writing, the article has received hundreds of citations. Of those citations, we only found two other studies that reported looking for the anterior cingulate cortex to CA1 projection as part of a wider study (Fillinger et al., 2018; Wang and Ikemoto, 2016); both publications briefly noted that they failed to find evidence of this projection.

One recent publication (Bian et al., 2019) did present evidence of a monosynaptic projection from anterior cingulate cortex to CA1 (both dorsal and ventral) using retrograde AAV tracing as part of a study into prefrontal-hippocampal circuitry in contextual fear memory. Interestingly, this study found a far more sparse projection than that reported earlier (Rajasethupathy et al., 2015), with figure 5 of the Bian study revealing comparable 'retrograde' labelling in both M2 and cingulate areas to our results showing off-target labelling with the rAAV-retro vector. In that same study (Bian et al., 2019) the viral vectors failed to evidence of a connection from vHPC to anterior cingulate areas, despite such inputs having been described with more traditional axonal tracers (e.g. Hoover and Vertes, 2007; Jay and Witter, 1991). Such discrepancies highlight the potential idiosyncrasies of different tracer techniques. 


\section{Stereotaxic coordinates and the borders of anterior cingulate cortex}

Prefrontal cortex has been extensively studied in multiple model organisms, from mice and rats to humans and nonhuman primates. However, despite a large amount of homology across species, the shape of the prefrontal areas in the brains of different species is quite varied. These variations in shape, together with distinctions in the cytoarchitecture of the subdivisions of the prefrontal cortex have led to a lack of agreed nomenclature and clear boundaries of specific prefrontal regions (see Laubach et al., 2018 for a comprehensive analysis of discrepancies between authors). In this paper, we use the nomenclature and delineation from Vogt \& Paxinos (Vogt and Paxinos, 2014) and the human homologies (Brodmann's areas). A very helpful online unified anatomical atlas, combining the Franklin-Paxinos and the common coordinate framework from the Allen Institute of Brain Science, was also used cross check the anatomical borders of the prefrontal areas (Chon et al., 2019).

The injection spread of the anterograde tracing experiment, shown on figure $1 \mathrm{~b}$ of the earlier study (Rajasethupathy et al., 2015) cleary includes the cingulate cortex, but also the M2 part of motor cortex is transduced by the virus; it is also possible that the transitional midcingulate area 24' (Vogt and Paxinos, 2014) has been involved. Indeed, when using the reported coordinates, we again found significant spread of the virus in M2. Furthermore, looking at the projection pattern of the anterograde fibres in Rajasethupathy et al. (2015), it can be noticed that both hippocampi show fluorescent fibres, with the fibres in the contralateral hippocampus (right; figure $1 \mathrm{~b}$ in their study) being brighter than in the ipsilateral hippocampus; the pattern, opposite to that in the case of the thalamus. Having used $250 \mathrm{nl}$ of virus, as compared with $500 \mathrm{nl}$ from Rajasethupathy et al. (2015) we still show the spillover neighbouring brain areas with these coordinates. Whilst the spillover of the virus itself cannot account for the apparent presence of axons from the cingulate cortex to CA1, nor the optogenetic activation of these afferents in that study, one potential explanation of a false positive could be that the relatively large volume of virus with a high titre 
injected close to the midline led to the leakage of virus beyond the intended injection site, resulting virus being taken up via axons traversing the cingulum bundle.

\section{$\underline{\text { Concluding remarks }}$}

Here we report that we have been unable to find evidence of a monosynaptic projection from anterior cingulate cortex to hippocampus proper in the mouse, replicating findings reported over decades of rodent neuroanatomical literature but in stark contrast to two recent reports (Bian et al., 2019; Rajasethupathy et al., 2015). While we have presented some tentative explanations that could account for these discrepancies, we remain unable to provide a full account of why we and others failed to find evidence of this projection, should it exist. Our intention in reporting our findings is not to criticise the research carried out by colleagues, but to initiate a wider conversation about how we use genetic tools in systems neuroscience, and interpret the data. The hippocampal - prefrontal system is perhaps one of the most widely studied long-range circuits in cognitive neuroscience; that controversies still exist on the precise cellular connections between these two areas serves to highlight both the excitement and challenges that arise as modern genetic tools provide us with opportunities to study neural circuitry in detail that was unimaginable just 20 years ago.

\section{Acknowledgements}

This work was supported by Biotechnology and Biological Sciences Research Council grant BB/P001475/1 (MTC). SY was a PhD student supported by Wellcome Trust grant 108891/B/15/Z. GMS and ESB were PhD students supported by grant MR/N0137941/1 for the GW4 BIOMED MRC DTP, awarded to the Universities of Bath, Bristol, Cardiff and Exeter from the Medical Research Council (MRC)/UKRI. Floxed TVA mice were kindly provided by Prof Dieter Saur at 
Technischen Universität München. We thank Dr E. Bubb and Dr A. Nelson (University of Cardiff)

for access to relevant tracer data.

\section{References}

Andrianova L, Brady ES, Margetts-Smith G, Kohli S, McBain CJ, Craig MT. 2021. Hippocampal CA1 pyramidal cells do not receive monosynaptic input from thalamic nucleus reuniens. doi:10.1101/2021.09.30.462517

Bian X-L, Qin C, Cai C-Y, Zhou Y, Tao Y, Lin Y-H, Wu H-Y, Chang L, Luo C-X, Zhu D-Y. 2019. Anterior Cingulate Cortex to Ventral Hippocampus Circuit Mediates Contextual Fear Generalization. J Neurosci 39:5728-5739. doi:10.1523/jneurosci.2739-18.2019

Cenquizca LA, Swanson LW. 2007. Spatial organization of direct hippocampal field CA1 axonal projections to the rest of the cerebral cortex. Brain Res Rev 56:1-26.

doi:10.1016/j.brainresrev.2007.05.002

Chon U, Vanselow DJ, Cheng KC, Kim Y. 2019. Enhanced and unified anatomical labeling for a common mouse brain atlas. Nat Commun 10:5067. doi:10.1038/s41467-019-13057-w

Eichenbaum H. 2017. Prefrontal-hippocampal interactions in episodic memory. Nat Rev Neurosci 18:547-558. doi:10.1038/nrn.2017.74

Fillinger C, Yalcin I, Barrot M, Veinante P. 2018. Efferents of anterior cingulate areas 24a and $24 \mathrm{~b}$ and midcingulate areas 24a' and 24b' in the mouse. Brain Struct Funct 223:1747-1778. doi:10.1007/s00429-017-1585-x

Furtak SC, Wei S, Agster KL, Burwell RD. 2007. Functional neuroanatomy of the parahippocampal region in the rat: The perirhinal and postrhinal cortices. Hippocampus 17:709-722. doi:10.1002/hipo.20314

Groenewegen HJ, Zee EV-V der, Kortschot A te, Witter MP. 1987. Organization of the projections from the subiculum to the ventral striatum in the rat. A study using anterograde transport of Phaseolus vulgaris leucoagglutinin. Neuroscience 23:103-120. doi:10.1016/0306-4522(87)90275-2

Guo H, Hong S, Jin X-L, Chen R-S, Avasthi PP, Tu Y-T, Ivanco TL, Li Y. 2000. Specificity and Efficiency of Cre-Mediated Recombination in Emx1-cre Knock-in Mice. Biochem Bioph Res Co 273:661-665. doi:10.1006/bbrc.2000.2870

Hoover WB, Vertes RP. 2007. Anatomical analysis of afferent projections to the medial prefrontal cortex in the rat. Brain Struct Funct 212:149-179. doi:10.1007/s00429-007-0150-4

Hurley KM, Herbert H, Moga MM, Saper CB. 1991. Efferent projections of the infralimbic cortex of the rat. J Comp Neurol 308:249-276. doi:10.1002/cne.903080210 
Jay TM, Witter MP. 1991. Distribution of hippocampal CA1 and subicular efferents in the prefrontal cortex of the rat studied by means of anterograde transport of Phaseolus vulgarisleucoagglutinin. J Comp Neurol 313:574-586. doi:10.1002/cne.903130404

Jones BF, Witter MP. 2007. Cingulate cortex projections to the parahippocampal region and hippocampal formation in the rat. Hippocampus 17:957-976. doi:10.1002/hipo.20330

Laubach M, Amarante LM, Swanson K, White SR. 2018. What, If Anything, Is Rodent Prefrontal Cortex? Eneuro 5:ENEURO.0315-18.2018. doi:10.1523/eneuro.0315-18.2018

Malik R, Li Y, Schamiloglu S, Sohal VS. 2021. Top-down control of hippocampal signal-to-noise by prefrontal long-range inhibition. Biorxiv 2021.03.01.433441. doi:10.1101/2021.03.01.433441

Mathiasen ML, O'Mara SM, Aggleton JP. 2020. The anterior thalamic nuclei and nucleus reuniens: So similar but so different. Neurosci Biobehav Rev 119:268-280. doi:10.1016/j.neubiorev.2020.10.006

Oh SW, Harris JA, Ng L, Winslow B, Cain N, Mihalas S, Wang Q, Lau C, Kuan L, Henry AM, Mortrud MT, Ouellette B, Nguyen TN, Sorensen SA, Slaughterbeck CR, Wakeman W, Li Y, Feng D, Ho A, Nicholas E, Hirokawa KE, Bohn P, Joines KM, Peng H, Hawrylycz MJ, Phillips JW, Hohmann JG, Wohnoutka P, Gerfen CR, Koch C, Bernard A, Dang C, Jones AR, Zeng H. 2014. A mesoscale connectome of the mouse brain. Nature 508:207-214. doi:10.1038/nature13186

Prasad JA, Chudasama Y. 2013. Viral Tracing Identifies Parallel Disynaptic Pathways to the Hippocampus. J Neurosci 33:8494-8503. doi:10.1523/jneurosci.5072-12.2013

Rajasethupathy P, Sankaran S, Marshel JH, Kim CK, Ferenczi E, Lee SY, Berndt A, Ramakrishnan C, Jaffe A, Lo M, Liston C, Deisseroth K. 2015. Projections from neocortex mediate top-down control of memory retrieval. Nature 526:653-659.

doi:10.1038/nature15389

Segal M, Landis S. 1974. Afferents to the hippocampus of the rat studied with the method of retrograde transport of horseradish peroxidase. Brain Res 78:1-15. doi:10.1016/00068993(74)90349-7

Seidler B, Schmidt A, Mayr U, Nakhai H, Schmid RM, Schneider G, Saur D. 2008. A Cre-loxPbased mouse model for conditional somatic gene expression and knockdown in vivo by using avian retroviral vectors. Proc National Acad Sci 105:10137-10142.

doi:10.1073/pnas.0800487105

Sesack SR, Deutch AY, Roth RH, Bunney BS. 1989. Topographical organization of the efferent projections of the medial prefrontal cortex in the rat: An anterograde tract-tracing study with Phaseolus vulgaris leucoagglutinin. J Comp Neurol 290:213-242.

doi:10.1002/cne.902900205

Sun Y, Nguyen AQ, Nguyen JP, Le L, Saur D, Choi J, Callaway EM, Xu X. 2014. Cell-TypeSpecific Circuit Connectivity of Hippocampal CA1 Revealed through Cre-Dependent Rabies Tracing. Cell Reports 7:269-280. doi:10.1016/j.celrep.2014.02.030 
Swanson LW. 1981. A direct projection from Ammon's horn to prefrontal cortex in the rat. Brain Res 217:150-154. doi:10.1016/0006-8993(81)90192-x

Tervo DGR, Hwang B-Y, Viswanathan S, Gaj T, Lavzin M, Ritola KD, Lindo S, Michael S, Kuleshova E, Ojala D, Huang C-C, Gerfen CR, Schiller J, Dudman JT, Hantman AW, Looger LL, Schaffer DV, Karpova AY. 2016. A Designer AAV Variant Permits Efficient Retrograde Access to Projection Neurons. Neuron 92:372-382. doi:10.1016/j.neuron.2016.09.021

Vogt BA, Miller MW. 1983. Cortical connections between rat cingulate cortex and visual, motor, and postsubicular cortices. J Comp Neurol 216:192-210. doi:10.1002/cne.902160207

Vogt BA, Paxinos G. 2014. Cytoarchitecture of mouse and rat cingulate cortex with human homologies. Brain Struct Funct 219:185-192. doi:10.1007/s00429-012-0493-3

Wang DV, Ikemoto S. 2016. Coordinated Interaction between Hippocampal Sharp-Wave Ripples and Anterior Cingulate Unit Activity. J Neurosci 36:10663-10672. doi:10.1523/jneurosci.1042-16.2016

Wickersham IR, Finke S, Conzelmann K-K, Callaway EM. 2007. Retrograde neuronal tracing with a deletion-mutant rabies virus. Nat Methods 4:47-49. doi:10.1038/nmeth999 